\title{
CYCLOTRON RESONANCE EFFECTS ON STOCHASTIC ACCELERATION OF LIGHT IONOSPHERIC IONS
}

\author{
Nagendra Singh, R.W. Schunk and J.J. Sojka \\ Center for Atmospheric and Space Sciences \\ Utah State University \\ Logan, Utah 84322
}

\begin{abstract}
Numerical studies on the interaction of electrostatic hydrogen cyclotron (EHC) waves with light ionospheric ions, such as $\mathrm{He}^{+}$, show that when the wave number ratio $\mathrm{k}_{\perp} / \mathrm{k}_{||} \leqslant 10$ resonant effects are important in the stochastic heating of the ions. When $k_{\perp} / k_{||}>10$ an appreciable acceleration occurs even though the cyclotron-resonant effects are absent. As the parallel wave number increases, causing $\mathrm{k}_{\perp} / \mathrm{k}_{||}$to decrease $(<10)$, the number of accelerated ions and the perpendicular and parallel energization increase.
\end{abstract}

\section{Introduction}

The production of energetic ions with conical pitch angle distributions along the auroral field lines is a subject of considerable current interest. Since the observations of conics from the ISIS 1 and 2 [Ungstrup et al, 1979; Klumpar, 1979] and S3-3 satellite and the observations of ion cyclotron and lower hybrid waves from the latter satellite [Kintner et al, 1978, 1979; Temerin et al, 1981], it is believed that the conics are the result of the acceleration of the ions transverse to the earth's magnetic field $\mathrm{B}$ and their subsequent upward expulsion due to the magnetic mirror force. As present, there are several theoretical treatments showing the acceleration (heating) of the ions by ion cyclotron waves [Palmadesso et al, 1974; Lysak et al, 1980; Papadopoulos et al, 1980; Dusenbery and Lyons, 1981; Singh et al, 1981] and by the lower hybrid resonance (LHR) waves [Chang and Coppi, 1981]. follows:

The typical wave parameters of an ion cyclotron wave are as

$$
\omega \cong \Omega_{\mathrm{i}}(1+\Delta), \mathrm{k}_{\perp} \cong \Omega_{\mathrm{i}} / \mathrm{V}_{\mathrm{Ti}}
$$

where $\omega$ and $\Omega_{i}$ are the wave and ion-cyclotron frequencies, $\Delta$ is typically $0.2, \mathrm{~V}_{\mathrm{Ti}}$ is the ion thermal speed and $\mathrm{k}_{\perp}$ is the wave vector component perpendicular to the magnetic field $B$. the quasilinear theory, in which the intereaction of ions with their respective ion cyclotron waves have been studied [Dusenbery and Lyons, 1981] predicts acceleration of ions only when they have cyclotron resonance with the waves. The cyclotron resonance velocity is given by

$$
\mathrm{V}_{\mathrm{i}} \cong \Delta\left(\mathrm{k}_{\perp} / \mathrm{k}_{||}\right) \mathrm{V}_{\mathrm{Ti}}
$$

where $k_{\|}$is the wave vector component parallel to $B$. For a typical value of $\Delta=0.2$, we note from (1) that $k_{\perp} / k_{\|} \leqslant 10$ for an appreciable number of ions to undergo acceleration. Otherwise, the resonance falls in a region of the velocity distribution function where there are very few ions. Thus, the quasilinear theory predicts no acceleration when the ions are non-resonant, which occurs for ion cyclotron waves having $\mathrm{k}_{1} / \mathrm{k}_{\|}>10$.

We demonstrate in this letter that the cyclotron resonances are not crucial for the transverse acceleration of ions by ion cyclotron waves. Transverse acceleration without such resonances can occur through stochastic acceleration [Karney, 1978; Papadopoulos et al, 1980; Singh et al, 1981]. Observations from the S3-3 satellite have indicated occurrences of strong

Copyright 1982 by the American Geophysical Union.

Paper number 2L1187.

0094-8276/82/002L-1187\$3.00 coherent Electrostatic Hydrogen Cyclotron (EHC) waves above an altitude of about $5000 \mathrm{~km}$ on auroral field lines [Kintner et al, 1978, 1979; Lysak et al, 1980; Temerin et al, 1981; Mizera et al, 1982]. Below about $5000 \mathrm{~km}$, the waves are broadband LHR waves; these waves are effective in transversely accelerating the ions [Chang and Coppi, 1981]. The EHC waves are strongly correlated with ion beams [Kintner et al, 1979], which are probably the result of a field-aligned potential drop [Temerin et al., 1981]. It has been demonstrated that $\mathrm{EHC}$ waves can energize the ionospheric ions, such as $\mathrm{He}^{++}, \mathrm{He}^{+}$, and $\mathrm{O}^{+}[$Papadopoulos et al, 1980; Singh et al, 1981]. Below, we show the effect of cyclotron resonances on the stochastic heating of the ionospheric ions by $\mathrm{EHC}$ waves.

Abe et al, [1980] have studied the effect of cyclotron resonances on the stochastic acceleration by varying the harmonic number $v$, which is an integer close to $\omega / \Omega(i)$, the ratio of wave frequency to the cyclotron frequency. If the wave is the EHC wave $\vartheta \sim \mathrm{M}(\mathrm{i}) / \mathrm{M}_{\mathrm{H}}$, where $\mathrm{M}_{\mathrm{H}}$ and $\mathrm{M}(\mathrm{i})$ are the masses of hydrogen ions and the ions interacting with the wave, respectively. Abe et al showed that when $v>10$, the cyclotron resonance width is exceedingly small, and hence, the number of resonant ions is also very small. Thus, the acceleration occurs through non-resonant stochastic heating. The theory for this acceleration mechanism was developed by Karney [1978] in the limit $\mathbf{k}_{\|}<<\mathbf{k}_{\perp}$. When $\vartheta<10$ there are an appreciable number of resonant ions. For $\mathrm{O}^{+}$ions $\vartheta \sim 16$, indicating resonant effects are not important, while for $\mathrm{He}^{+}$ions $v \sim 4$ and the possibility exists for strong resonant effects if the parallel resonant velocity falls in the bulk of the initial distribution.

\section{Numerical Results}

We have studied numerically the interactions of ionospheric ions and a coherent wave, given by

$$
\phi=\phi_{0} \cos \left(\mathrm{k}_{\perp} \mathrm{X}+\mathrm{k}_{||} \mathrm{Z}-\omega \mathrm{t}\right)
$$

where $\phi$ is the wave potential and $\phi_{0}$ is its amplitude. The equations of motion of 2000 ions were solved as a function of time. Details of the numerical technique are described by Singh et al, [1981] and are not repeated here. In the present study, we consider a hydrogen cyclotron wave with typical parameters

$$
\hat{\phi}_{\mathrm{o}}=\mathrm{e} \phi_{\mathrm{o}} / \mathrm{k}_{\mathrm{B}} \mathrm{T}_{\mathrm{H}}=0.4, \mathrm{k}_{\perp} \mathrm{r}_{\mathrm{H}}=1.7, \frac{\omega}{\Omega_{\mathrm{H}}}=1.2
$$

where $k_{B}$ is Boltzmann's constant. The quantities $T_{H}, r_{H}$ $\left[=\left(k_{B} T_{H} / M_{H}\right)^{1 / 2} / \Omega_{H}\right]$, and $\Omega_{H}$ are the temperature, Larmor radius and cyclotron frequency for the hydrogen ions, respectively. The wave number ratio $k_{\perp} / k_{||}$was varied from 3 to 100 . In the following discussion we present results for $\mathrm{He}^{+}$ions, which were assumed to be minor ions.

Figures 1(a) to (d) show $\mathrm{He}^{+}$velocity distribution functions in the $V_{\| I}-V_{\perp}$ plane for several $k_{\perp} / k_{\|}$values. The velocities are normalized with respect to the initial ion thermal speed, $\mathrm{a}\left(\mathrm{He}^{+}\right)$ $=\left[2 \mathrm{k}_{\mathrm{B}} \mathrm{T}\left(\mathrm{He}^{+}\right) / \mathrm{M}\left(\mathrm{He}^{+}\right)\right] \cdot{ }^{1 / 2}$ The initial $\mathrm{He}^{+}$temperature was chosen to be $T\left(\mathrm{He}^{+}\right)=\mathrm{T}_{\mathrm{H}} / 10$. For this case, appreciable heating occurred [Singh et al, 1981]. The contours shown in the figures are for constant phase-space densities $\mathrm{N}$. The numbers on the contours indicate the density in units of $\ln (\mathrm{N})=1 / 2$. The resonance velocities for $v=4$ and 5 are shown for the cases where cyclotron resonances seem to affect the ion dynamics. 


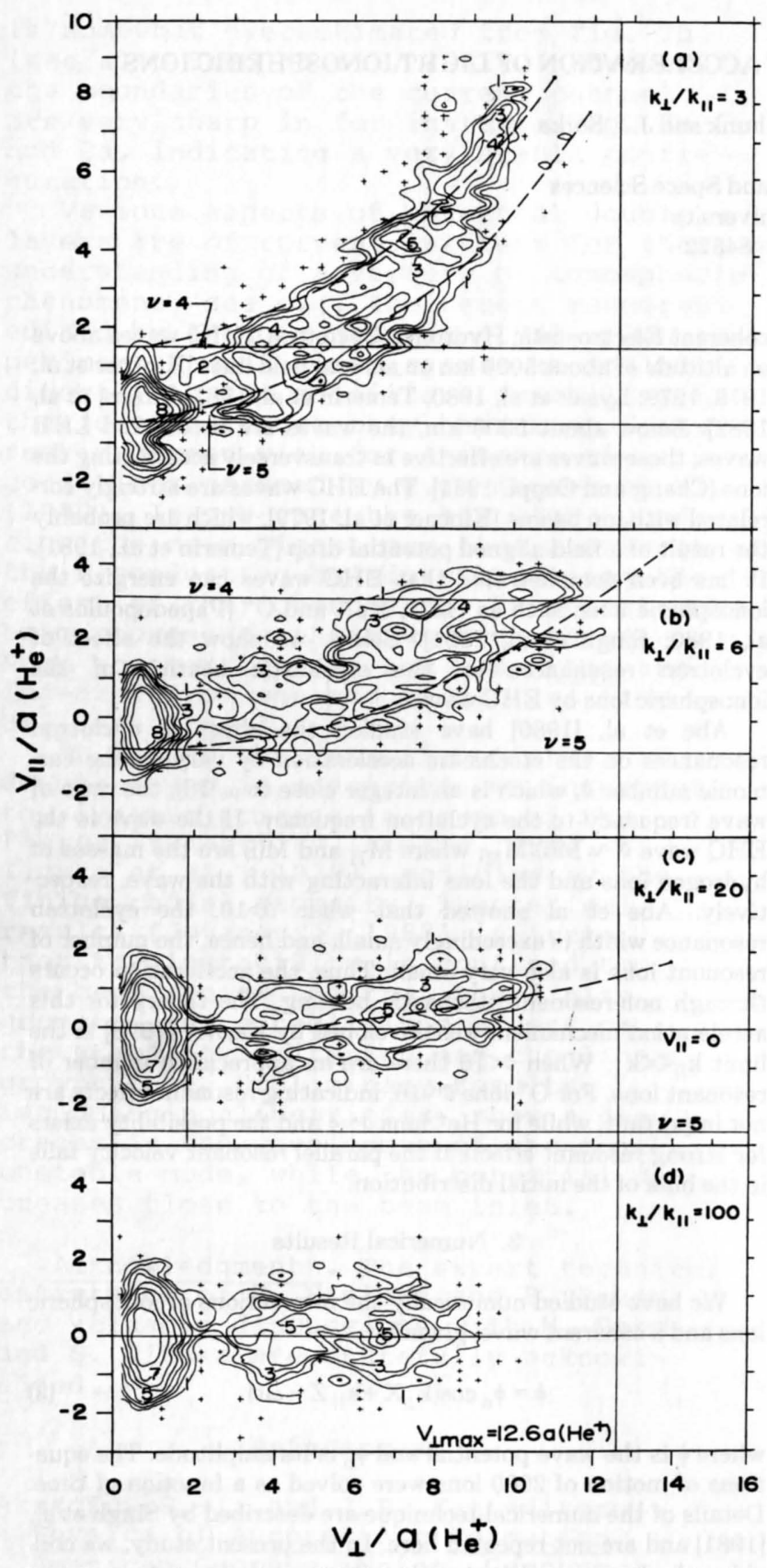

Figure 1. $\mathrm{He}^{+}$velocity distribution functions in the $\mathrm{V}_{\|}-\mathrm{V}_{\perp}$ plane for several values of $k_{\perp} / k_{\| \mid}$. The horizontal lines marked with $v=4$ and 5 indicate the cyclotron resonant parallel valocity of the ions.

When $\mathbf{k}_{\perp} / \mathbf{k}_{\|}=3$, the resonances fall within the bulk, as shown by the horizontal lines marked $v=4$ and 5 . When $\mathrm{k}_{\perp} / \mathrm{k}_{||}=6$, only the $v=5$ resonance falls in the bulk of the initial distribution. When $k_{1} / k_{||}=20$ and 100 , the resonances fall far from the bulk, where there are hardly any ions. Despite these significant differences. Figures 1(a) to (d) show that appreciable acceleration occurs for all 4 cases. However, the details of the acceleration critically depend on the ratio $\mathbf{k}_{\perp} / \mathbf{k}_{\|}$. Both the parallel and perpendicular accelerations are the smallest when $k_{\perp} / k_{1 \mid}=100$; this case closely approximating the theory of Karney [1978]. As $\mathbf{k}_{\|}$increases, the parallel and perpendicular accelerations increase. Note that $k_{\perp} / k_{||}=100$ may be too large a value for the EHC wave. However, this closely illustrates the non-resonant acceleration with $\mathbf{k}_{||}=0$.
In order to understand the acceleration process quantita. tively, we give a brief description of the stochastic acceleration of ions by a coherent wave. In the presence of such a wave, the acceleration of ions proceeds as long as the following threshold criterion is met [Fakuyama et al, 1977; Abe et al, 1980; Karney, 1981],

$$
\begin{gathered}
\delta=v \alpha\left|J_{\vartheta}\left(\xi_{\mathrm{p} \vartheta}\right)\right| / \xi_{\mathrm{p} v} \gtrsim \frac{1}{4}, \mathrm{p}=1,2,3,4 \ldots \\
\alpha=\mathrm{k}_{\perp}^{2} \mathrm{q} \phi_{\delta} / \mathrm{M}_{\mathrm{i}} \Omega_{\mathrm{i}}^{2}
\end{gathered}
$$

where $M_{i}$ and $\Omega_{i}$ are the mass and cyclotron frequency of the ions undergoing acceleration. $J_{\vartheta}(\xi)$ is the Bessel function of order $v$, where $v$ is an integer close to $\omega / \Omega_{\mathrm{i}} . \xi=\mathbf{k}_{\perp} r_{\mathrm{i}}$, where $\mathrm{r}_{\mathrm{i}}$ is the Larmor radius defined by $r_{i}=V_{\perp} / \Omega_{i}, V_{\perp}$ being the perpendicular velocity of the ion. $\xi_{p} v$ is determined by $[\mathrm{dJ} v(\xi) / \mathrm{d} \xi]_{\xi}=\xi_{\mathrm{pv}}=$ 0 . The ranges of $\xi$ separated by the zero of $J_{v}(\xi)$ are called cells. The index $p$ on $\xi$ indicates the cell number beginning at the lowest cell.

Using the wave parameters in (4), we note that $v=5$ and $\alpha \cong$ 4.6, and the threshold is met up to the fourth cell, in which $\delta \cong$ 0.24 . The upper boundary of this cell corresponding to the zero of $J_{5}\left(k_{\perp} r\right)$ gives the maximum value of $r_{i}$

$$
\mathrm{k}_{\perp} \mathrm{r}_{\text {imax }} \cong 19 \text { or } \mathrm{V}_{\perp \max } \cong 12.6 \mathrm{a}\left(\mathrm{He}^{+}\right)
$$

This velocity is indicated in Figures $1(\mathrm{a})$ to (d) by the vertical solid line. Thus, equation (7) predicts a maximum perpendicular energization of $\mathrm{E}_{\max }=1 / 2 \mathrm{M}\left(\mathrm{He}^{+}\right) \mathrm{V}_{\perp \max }^{2} \cong 160 \mathrm{~K}_{\mathrm{B}} \mathrm{T}\left(\mathrm{He}^{+}\right)=16$ $k_{B} T_{H}$. Such a heating is seen in Figure 1 when $k_{\perp} / k_{\|} z 6$. When $\mathbf{k}_{\perp} / \mathbf{k}_{||}=100, \mathrm{~V}_{\perp \max }=10 \mathrm{a}\left(\mathrm{He}^{+}\right)$, giving $\mathrm{E}_{\max }=10 \mathrm{k}_{\mathrm{b}} \mathrm{T}_{\mathrm{H}}$, which is in agreement with Papadoupoulos et al [1980]. Note that the basic difference between our estimate in (7) and that of Papadopoulos et al is due to the resonance effects; in deriving their heating estimates these authors replaced $v$ by $\mathrm{M}(\mathrm{i}) / \mathrm{M}_{\mathrm{H}}$, giving the mass dependence of the maximum heating as $\mathrm{E}_{\max }$ $\sim\left(\mathrm{M}(\mathrm{i}) / \mathrm{M}_{\mathrm{H}}\right)^{5 / 3}$. When resonance effects are present, the use of $\vartheta=5$ accounts for the larger heating predicted in (7).

In order to understand the parallel acceleration, we recall that in the wave frame the total energy of an ion remains constant

$$
\frac{1}{2} M_{i}\left[\left(V_{\|}-\omega / k_{\|}\right)^{2}+V_{I}^{2}\right]+q \phi=\text { const. }
$$

When the changes in velocity are such that $\Delta V^{2} \gg 2 q \phi / M_{i}$ and when $\omega / k_{||} \gg V_{||}$, (8) can be written as

$$
\Delta \mathrm{V}_{\|} \cong \frac{\mathbf{k}_{\|}}{2 \omega} \Delta \mathrm{V}_{\perp}^{2}
$$

The parabolic curves in Figures 1(a) to (c) are given by (9). Note that the accelerations follow the parabolic curves quite closely.

To summarize the discussion to this point, we note that irrespective of the value of $k_{\perp} / k_{\|}$, large transverse accelerations occur accompanied by a parallel acceleration as given by (9). We show below that the number of accelerated ions is significant even when there are no cyclotron resonances due to a small parallel wave number.

Using the criterion that any ion falling in the tail $\mathrm{V}>2$ $\mathrm{a}\left(\mathrm{He}^{+}\right)$, is energetic, we found 1174 out of $2000(\sim 60 \%)$ energetic ions when $k_{\perp} / k_{||}=3$. When $k_{\perp} / k_{||}=6$, the percentage of energetic ions was about $46 \%$. Finally, when $k_{\perp} k_{\|} \gtrsim 20$, the number of accelerated ions was nearly constant at about $36 \%$ of the initial population.

In Figures 2(a) and (b) we compare the initial source population which undergoes acceleration for $k_{\perp} / k_{\|}=3$ and 100. The noteworthy feature of the contour plots is the sharp cut-off for small $V_{\perp}$ when $k_{\perp} / k_{\|}=100$. Such a sharp cut-off is not seen when $k_{\perp} / k_{||}=3$, for which a large number of particles come from the region of the overlapping resonances $\vartheta=4$ and 5 , as indicated by the parallel resonance velocities marked by the arrows. In the limit of $\mathrm{k}_{||}{ }^{\circ}$, the minimum perpendicular velocity $\mathrm{V}_{\perp 0}$. 


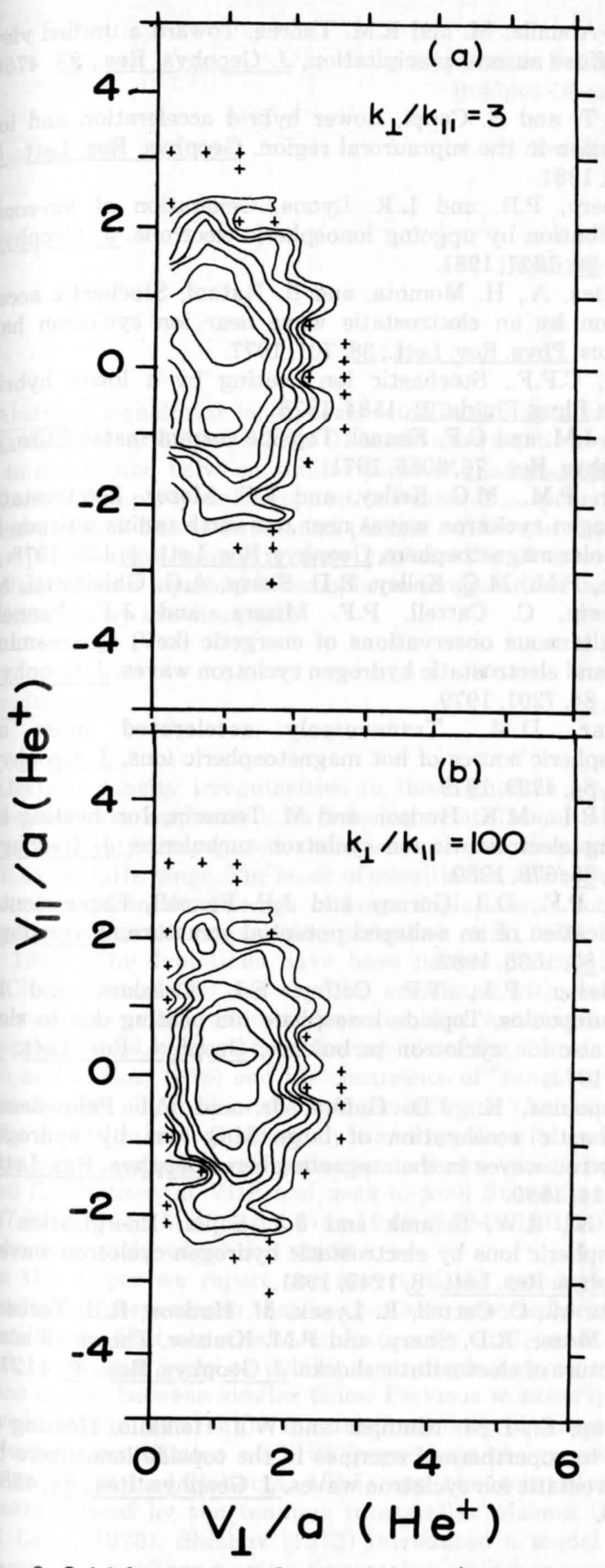

Figure 2. Initial source population of the $\mathrm{He}^{+}$ions which eventually underwent acceleration and ended up with $\mathrm{V}>2 \mathrm{a}\left(\mathrm{He}^{+}\right)$. The arrows in (a) indicate the parallel resonance velocities corresponding to $v=5$ (lower) and $v=4$ (upper). Note the sharp cutoff of contours at low perpendicular velocities in (b).

above which stochastic acceleration is possible, was estimated by Karney [1978] to be

$$
\mathrm{V}_{\perp 0} \cong \frac{\omega}{\mathrm{k}_{\perp}}\left(1-\alpha^{1 / 2} / v\right)
$$

For the parameters used here for $\mathrm{He}^{+}$ions, $\mathrm{V}_{\perp 0} \cong 1.8 \mathrm{a}\left(\mathrm{He}^{+}\right)$. Our numerical results show that this is an overestimate for the minimum value of the perpendicular velocity for acceleration. From Figure 2(b) we observe that $V_{10} \cong a\left(\mathrm{He}^{+}\right)$. An estimate of the number of particles $\mathrm{N}_{\mathrm{a}}$ undergoing acceleration can be obtained from a simple calculation

$$
\begin{gathered}
\mathrm{N}_{\mathrm{a}}=\mathrm{N}_{\mathrm{o}} \frac{2}{\sqrt{\pi}} \frac{1}{\mathrm{a}^{3}\left(\mathrm{He}^{+}\right)} \int_{-\infty}^{\infty} \mathrm{dV}_{\|} \int_{\mathrm{V}_{\perp 0}}^{\infty} \mathrm{dV}_{\perp} \mathrm{V}_{\mathrm{V} \perp} \exp \left[-\left(\mathrm{V}_{\|}{ }^{2}+\mathrm{V}_{\perp}{ }^{2}\right) / \mathrm{a}^{2}\left(\mathrm{He}^{+}\right)\right] \\
=\mathrm{N}_{\mathrm{o}} \exp \left[-\mathrm{V}_{\perp 0} / \mathrm{a}(\mathrm{He})\right]
\end{gathered}
$$

where $\left.\mathrm{N}_{0} \cong 2000\right)$ is the total number of ions. With $\mathrm{V}_{\perp 0} \cong$ $\mathrm{a}\left(\mathrm{He}^{+}\right),(11)$ gives $37 \%$ of the ions in the energetic tail, in close agreement with the numerical result.

We note that our numerical calculation has shown conclusively that a non-resonant stochastic acceleration occurs for low harmonic values of $\vartheta$. Thus, EHC waves, as observed by Kintner et al [1978, 1979], should be capable of creating conics in the pitch angle distribution of both light $\left(\mathrm{He}^{+}\right)$as well as heavy $\left(0^{+}\right)$ions even when the resonant effects are absent.

\section{Pitch Angle Distributions}

In experimental situations, very often the ion distributions are presented as pitch angle versus energy distributions of the differential number flux. Figures 3(a) and (b) show such plots for $\mathbf{k}_{\perp} / \mathbf{k}_{\|}=3$ and 100 . When $\mathbf{k}_{\perp} / \mathbf{k}_{\|}=100$, the maximum $\mathrm{He}^{+}$energy is about 100 times the initial $\mathrm{He}^{+}$temperature or $10 \mathrm{~T}_{\mathrm{H}}$. This is in agreement with Papadopoulos et al [1980]. On the other hand, for $\mathbf{k}_{\perp} / \mathbf{k}_{||}=3$, the maximum energy gain is almost double that value, $20 \mathrm{~T}_{\mathrm{H}}$. Thus, when the parallel wave number is large, the ions can extract a larger energy from the wave than when $\mathrm{k}_{\|}=$ 0 . The contributions to the larger energization come from both the parallel and perpendicular acceleration. However, if $\mathrm{H}^{+}$ temperatures are of the order of $1 \mathrm{eV}$, the maximum energy expected for $\mathrm{He}^{+}$can be only about $20 \mathrm{eV}$, which may make the detection of accelerated $\mathrm{He}^{+}$ions difficult.

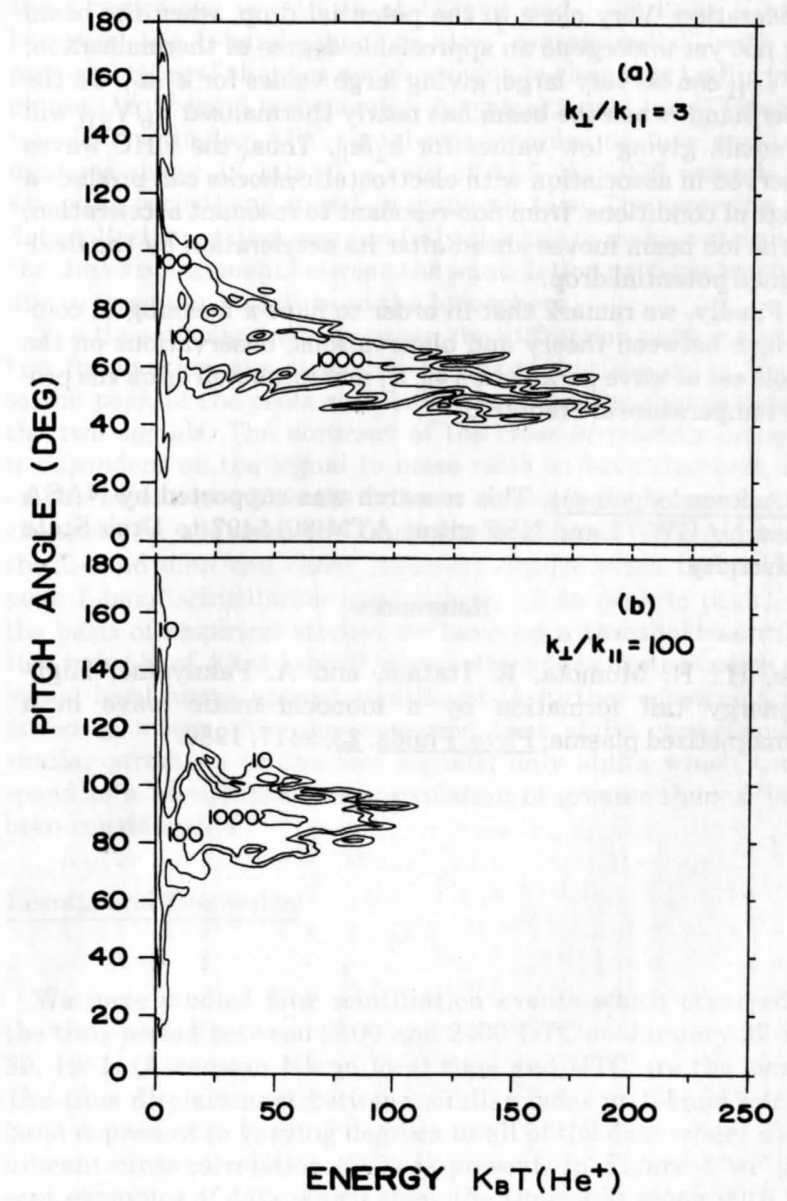

Figure 3. Contour plots in the pitch angle versus energy plane for the two extreme values of $k_{\perp} / k_{||}$considered. 
The acceleration for $k_{\perp} / k_{\|}>20$ generates nearly a pancake distribution for the energetic particles, while for small values of $k_{\perp} / k_{||}$conics could be generated locally. For $k_{\perp} / k_{||}=3$, the minimum pitch angle is about $45^{\circ}$ at the maximum energy. The pitch angle decreases with energy, as predicted by (9).

\section{Summary and Discussion}

We conclude by noting that transverse energization of ionospheric ions, such as $\mathrm{He}^{+}, \mathrm{He}^{++}, \mathrm{O}^{++}$and $\mathrm{O}^{+}$, are possible by an EHC wave even in the absence of cyclotron resonance. The mechanism of acceleration is the non-resonant stochastic heating. However, when there are resonant ions both the total energy gain and the number of accelerated ions increase with increasing parallel wave number.

Electrostatic ion cyclotron waves can be driven either by an electron current or an ion beam. The excitation of these waves by field-aligned currents in the topside ionosphere has been demonstrated by Kindel and Kennel [1971] and Ashour-Abdalla and Thorne [1978]. The ratio $\mathrm{k}_{\perp} / \mathrm{k}_{||}$may range from 5 to 10 in the topside ionosphere as the proton to electron temperature ratio varies from a very large value to unity. Thus, strong resonant effects for the light ions are expected in the topside ionosphere.

EHC waves observed in association with electrostatic shocks at altitudes of about one earth radius are found to have strong correlations with ion beams accelerated by field-aligned potential drops [Kintner et al, 1979; Temerin et al, 1981; Mizera et al, 1982]. This suggests that the waves are excited by the beam. In such a situation, the expected values of $\mathbf{k}_{\perp} / \mathbf{k}_{\|} \sim$ $\mathrm{V}_{\mathrm{b}} / \mathrm{V}_{\mathrm{TH}}$, where $\mathrm{V}_{\mathrm{b}}$ and $\mathrm{V}_{\mathrm{TH}}$ are the ion beam velocity and its thermal speed. The ratio $\mathrm{V}_{\mathrm{b}} / \mathrm{V}_{\mathrm{TH}}$ will be dependent on the fieldaligned potential drop and on the evolution of the beam after acceleration. Very close to the potential drop, when the beam has not yet undergone an appreciable degree of thermalization, $\mathrm{V}_{\mathrm{b}} / \mathrm{V}_{\mathrm{TH}}$ can be very large, giving large values for $\mathrm{k}_{\perp} / \mathrm{k}_{\|}$. On the other hand, when the beam has nearly thermalized $\mathrm{V}_{\mathrm{b}} / \mathrm{V}_{\mathrm{TH}}$ will be small, giving low values for $\mathbf{k}_{\perp} / \mathbf{k}_{\| \mid}$. Thus, the EHC waves observed in association with electrostatic shocks can produce a range of conditions, from non-resonant to resonant acceleration, as the ion beam moves ahead after its acceleration by the fieldaligned potential drop.

Finally, we remark that in order to have a meaningful comparison between theory and observations, observations on the whole set of wave parameters $\phi_{0}, \mathrm{k}_{\perp}$ and $\mathrm{k}_{||}$as well as on the proton temperature are required.

Acknowledgement. This research was supported by NASA grant NAGW-77 and NSF grant ATM-8015497 to Utah State University.

\section{References}

Abe, H., H. Momota, R. Itatani, and A. Fakuyama, Highenergy tail formation by a monochromatic wave in a magnetized plasma, Phys. Fluids, $\underline{\text { 23, }}$ 2417, 1980.
Ashour-Abdalla, M. and R.M. Thorne, Toward a unified view of diffuse auroral precipitation, J. Geophys. Res., $\underline{83}, 4755$, 1978.

Chang, T. and B. Coppi, Lower hybrid acceleration and ion evolution in the suprauroral region, Geophys. Res. Lett., $\underline{8}$, 1253, 1981.

Dusenbery, P.B. and L.R. Lyons, Generation of ion-conic distribution by upgoing ionospheric electrons, J. Geophys. Res., $\underline{86}, 7627,1981$.

Fakuyama, A., H. Momota, and R. Itatani, Stochastic acceleration by an electrostatic wave near ion cyclotron harmonics, Phys. Rev. Lett., $\underline{\text { 38, 701, } 1977 .}$

Karney, C.F.F., Stochastic ion heating by a lower hybrid wave, Phys. Fluids, 21, 1584, 1978.

Kindel, J.M. and C.F. Kennel, Topside current instabilities, J. Geophys. Res., 76, 3055, 1971.

Kintner, P.M., M.C. Kelley, and F.S. Mozer, Electrostatic hydrogen cyclotron waves near one earth radius altitude in the polar magnetosphere, Geophys. Res. Lett., 5, 139, 1978.

Kintner, P.M., M.C. Kelley, R.D. Sharp, A.G. Ghielmetti, M. Temerin, C. Cattell, P.F. Mizera, and J.F. Fennell, Simultaneous observations of energetic (keV) upstreaming ions and electrostatic hydrogen cyclotron waves, J. Geophys. Res., 84, 7201, 1979.

Klumpar, D.M., Transversely accelerated ions: an ionospheric source of hot magnetospheric ions, J. Geophys. Res., 84, 4229, 1979.

Lysak, R.L., M.K. Hudson and M. Temerin, Ion heating by strong electrostatic ion cyclotron turbulence, J. Geophys. Res., 85, 678, 1980.

Mizera, P.F., D.J. Gorney and J.F. Fennell, Experimental verification of an s-shaped potential structure, J. Geophys. Res., $\underline{87}, 1535,1982$.

Palmadesso, P.J., T.P. Coffey, S.L. Ossakow and K. Papadopoulos, Topside ionosphere ion heating due to electrostatic ion cyclotron turbulence, Geophys. Res. Lett., 1 , $105,1974$.

Papadopoulos, K., J.D. Gaffey, Jr. and P.J. Palmadesso, Stochastic acceleration of large $M / Q$ ions by hydrogen cyclotron waves in the magnetosphere, Geophys. Res. Lett., $7,1014,1980$.

Singh, N., R.W. Schunk and J.J. Sojka, Energization of ionospheric ions by electrostatic hydrogen cyclotron waves, Geophys. Res. Lett., 8, 1249, 1981.

Temerin, M., C. Cattell, R. Lysak, M. Hudson, R.B. Torbert, F.S. Mozer, R.D. Sharp, and P.M. Kintner, The small scale structure of electrostatic shocks, J. Geophys. Res., 트, 11278, 1981.

Ungstrup, E., D.M. Klumpar and W.J. Heikkila, Heating of ions to superthermal energies in the topside ionosphere by electrostatic ion cyclotron waves, J. Geophys. Res., $\underline{84}, 4289$, 1979.
(Received May 14, 1982; accepted July 30, 1982.) 